\title{
Critical Effect of Detergent:Protein Ratio on the Formation of Hepatitis C Virus p7 Channel
}

\author{
Wen Chen ${ }^{\S}$, Bo OuYang ${ }^{\dagger}$, James J. Chou ${ }^{\S}$ \\ §Department of Biological Chemistry and Molecular Pharmacology, Harvard Medical School, \\ Boston, Massachusetts 02115, USA. \\ †State Key Laboratory of Molecular Biology, CAS Center for Excellence in Molecular Cell Science, \\ Shanghai Institute of Biochemistry and Cell Biology, Chinese Academy of Sciences, University of \\ Chinese Academy of Sciences, Shanghai 201203, China
}

\section{Abstract}

The $\mathrm{p} 7$ protein encoded by the Hepatitis $\mathrm{C}$ virus forms a cation-selective viroporin in membrane. One of the most intriguing findings about the $\mathrm{p} 7$ viroporin is its unique hexameric structure in dodecyl-phosphocholine (DPC) micelles determined by NMR. But, the hexameric structure was recently challenged by another NMR study of $\mathrm{p} 7$, also in DPC detergent, which claimed that the p7 in this detergent is monomeric. Here, we show that $p 7$ oligomerization is highly sensitive to the detergent:protein ratio used in protein reconstitution, and that the 40 -fold difference in this ratio between the two studies was the cause of their different conclusions. Further, we have performed extensive measurements of inter-chain paramagnetic relaxation enhancements (PREs) for $\mathrm{p} 7$ hexamers reconstituted in DPC micelles and in DMPC-DHPC bicelles. In both cases, inter-chain PREs are overall consistent with the hexameric structure determined in micelles. Our data validate the overall architecture of the p7 hexamer while highlighting the importance of detergent:protein ratio in membrane protein sample preparation.

\section{Graphical Abstract}

SYNOPSIS TOC: The oligomerization of the viral membrane protein $\mathrm{p} 7$ from Hepatitis $\mathrm{C}$ virus has been a matter of intense debate. This study discovers that $\mathrm{p} 7$ oligomerization is highly sensitive to the detergent:protein ratio used in protein reconstitution. Extensive measurements of inter-chain paramagnetic relaxation enhancements indicate that the hexameric conformations of $\mathrm{p} 7$ in bicelles and micelles are similar.

Corresponding Author: Correspondence should be addressed to james_chou@hms.harvard.edu. Author Contributions

W.C., B.O., J.J.C. conceived the study; W.C. prepared the samples and conducted experiments. W.C., B.O., J.J.C. wrote the paper. The Supporting Information is available free of charge on the ACS Publications website. File type: PDF) Detailed method description and Figures S1-S8. 


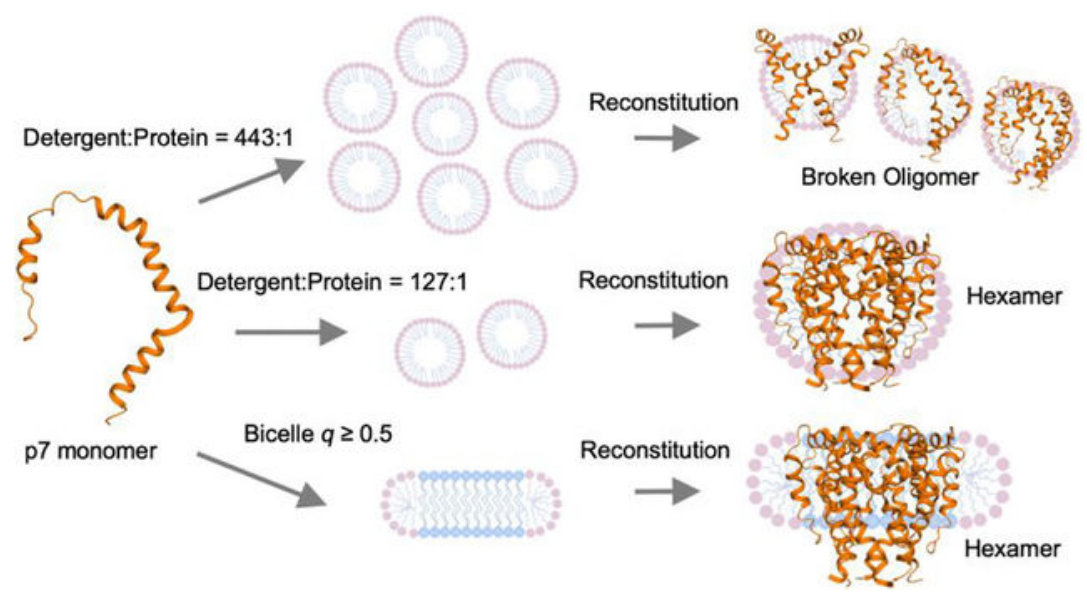

\section{Keywords}

detergent:protein ratio; size-exclusion chromatography; bicelle; NMR spectroscopy

The viroporin protein $\mathrm{p} 7$ encoded by the Hepatitis $\mathrm{C}$ virus (HCV) genome is a 63-residue protein that oligomerizes in membrane to form cation-selective channels ${ }^{1,2}$, with higher selectivity for $\mathrm{Ca}^{2+}$ than $\mathrm{K}^{+} / \mathrm{Na}^{+3}$, 4 . The channel activity of $\mathrm{p} 7$ is important for the assembly and release of infectious viruses ${ }^{5,6}$, and thus finding small molecules to block the $\mathrm{p} 7$ is an alternative route of developing therapeutics for treating $\mathrm{HCV}$ infection ${ }^{1,3,4,7}$.

As in the cases of influenza M2 channels, structural characterization of $\mathrm{p} 7$ was confronted with various technical problems; the hydrophobic and dynamic nature of the protein resisted crystallization, and the small molecular mass still poses serious hurdle for cryo-electron microscopy (cryo-EM). In a previous study to enable solution nuclear magnetic resonance (NMR) measurements, we developed a sample of the p7 from genotype 5a (EUH1480 strain), designated p7(5a), reconstituted in dodecyl-phosphocholine (DPC) micelles ${ }^{8}$. Negative-stain electron microscopy (nsEM) analysis of that sample generated 2D referencefree class averages that are indicative of hexamers and that resemble the nsEM images of p7(2a) (JFH-1 strain) in the $\mathrm{DH}_{7} \mathrm{PC}$ detergent ${ }^{9}$. Further NMR analysis of both intra- and inter- chain nuclear Overhauser effects (NOEs) led to a detailed model of the p7(5a) hexameric complex ${ }^{8,10}$.

The NMR structure shows that although $\mathrm{p} 7$ is only a $7 \mathrm{kDa}$ protein, it forms a rather sophisticated hexameric, funnel-like architecture with six intertwined chains ${ }^{8}$. Each chain contains three helical segments, designated $\mathrm{H} 1, \mathrm{H} 2$ and $\mathrm{H} 3$. The $\mathrm{H} 1$ and $\mathrm{H} 2$ helices form the narrow and wide regions of the funnel-shaped cavity, respectively, and the $\mathrm{H} 3$ helices wrap the channel periphery by interacting with $\mathrm{H} 2$ of the $\mathrm{i}+2$ and $\mathrm{H} 1$ of the $\mathrm{i}+3$ protomers. The high degree of inter-chain interactions allow a small peptide to form a $42 \mathrm{kDa}$ channel complex.

The intriguing hexameric structure of $\mathrm{p} 7$ was, however, challenged recently by studies by Oestringer et al, which argued that the $\mathrm{p} 7$ reconstituted in DPC detergent was monomeric ${ }^{11,12}$. The authors of the studies also suggested that the hexamer structure of the 
p7 was an artifact generated using inter-chain structural restraints that did not exist. Since the oligomeric architecture of $\mathrm{p} 7$ represents only the second type of viroporin structures after that of the Influenza M2 channels, the newly raised controversy was a major setback for the field of viroporin.

In this study, we performed experiments to understand why the DPC-reconstituted samples used by Oestringer et al and us resulted in completely different observations. We found that the detergent:protein ratio used during $\mathrm{p} 7$ reconstitution has a strong influence on the oligomeric assembly of p7(5a), i.e., the hexamer dissociates as the DPC:protein ratio increases, and thus the very different DPC:protein ratio used by the two studies was the cause of the major discrepancy. Further, we have performed extensive inter-chain paramagnetic relaxation enhancement (PRE) analyses of p7(5a) in DPC micelles and in DMPC-DHPC bicelles that closely mimic a lipid bilayer. In both cases, the data are consistent with the published hexameric structure of $\mathrm{p} 7(5 \mathrm{a})$.

We prepared p7(5a) samples (UniProt ID: 039928) with different amount of DPC to examine the effect of detergent:protein molar ratios on $\mathrm{p} 7$ oligomerization. The $\mathrm{p} 7(5 \mathrm{a})$ protein was purified and reconstituted in DPC as described previously ${ }^{8,13}$, except size-exclusion chromatography (SEC) was not applied. The samples with various DPC:p7 ratios were analyzed by SEC using the superdex 200 increase 10/30 column. Although the same elution buffer containing $3 \mathrm{mM}$ DPC was used, samples reconstituted originally with different DPC:p7 ratios showed very different elution profiles. When DPC:p7 molar ratio was 127:1, p7 eluted at $\sim 14 \mathrm{ml}$ as a single specie (Fig. 1a), indicative of a homogenous hexameric complex observed previously by nsEM. When DPC:p7 ratio was increased to 443:1, p7 eluted as two species: a minor peak at $\sim 14 \mathrm{ml}$ and the major broader peak at $\sim 16 \mathrm{ml}$ (Fig. $1 b)$, indicating that the hexameric complex has largely dissociated to lower, less homogeneous oligomers. When DPC:p7 ratio was further increased to 1194:1, the p7 elution peak at $\sim 14 \mathrm{ml}$ moved almost entirely to $\sim 16 \mathrm{ml}$ (Fig. 1c), indicating that the hexamer complex has completely dissociated to lower oligomers. Finally, at DPC:p7 ratio of 10,000:1, the elution peak shifted further to the right while becoming extremely broad, indicative of even more heterogeneous species of which the dominant specie is probably monomeric.

In the study by Oestringer et al ${ }^{12}$, a DPC:p7 ratio of 10,000 (5 $\mu \mathrm{M}$ p7(5a) and $50 \mathrm{mM}$ DPC) was used in the same reconstitution protocol as the one used in our current study. For this sample, SEC elution volume from the superdex 200 increase $10 / 30$ column was $18.7 \mathrm{ml}^{12}$, consistent with our result in Fig. 1d. The SEC data collectively show that oligomerization of p7(5a) is highly sensitive to the DPC:protein ratio, and the $\sim 40$ fold higher DPC:protein ratio used by Oestringer et al than that in our previous structural study explains why they only observed 7 monomers in their SEC analysis ${ }^{12}$.

We next performed a qualitative evaluation of the p7 hexamer structures in DPC micelles and in the more membrane-like DMPC-DHPC bicelles by measuring inter-chain PREs for the two reconstitution systems. In earlier study, we developed an NMR-feasible sample of $\mathrm{p} 7$ (5a) in DMPC-DHPC bicelles with $q=0.5$, showing that $\mathrm{p} 7$ (5a) also forms hexamer in 
bicelles ${ }^{14}$. The current study takes a step further to qualitatively address the oligomeric arrangement in bicelles relative to the hexameric structure in micelles.

For measuring inter-chain PREs, the NMR-visible, $\left({ }^{15} \mathrm{~N}, 80 \%{ }^{2} \mathrm{H}\right)$-labeled chain was mixed with the NMR-invisible, spin-labeled chain at 1:1 ratio, such that the detected PREs are exclusively inter-molecular. Each spin-labeled chain contains a single mutation to cysteine for labeling with MTSL (1-oxyl-2,2,5,5-tetramethyl- $\Delta 3$-pyrroline-3-methyl methanethiosulfonate). Inter-chain PREs were examined for four different single-site MTSL labels at residue positions $1,31,44$, and 57 , respectively. The $\left({ }^{15} \mathrm{~N}, 80 \%{ }^{2} \mathrm{H}\right)$ - and spinlabeled chains were mixed in organic solvent, followed by reconstitution in DPC and MTSL labeling (Supplementary Methods, NMR sample preparation). DPC:protein ratio for the PRE samples was $\sim 75$. Residue-specific PREs were measured as the ratio of NMR peak intensities in the ${ }^{1} \mathrm{H}_{-}{ }^{15} \mathrm{~N}$ TROSY-HSQC spectra (Figs. S1-S4) before (I) and after $\left(\mathrm{I}_{0}\right)$ reducing the samples with ascorbate.

The strongest inter-chain PREs generated by the 4 spin labels range from 0.3 to 0.7 , expected of 1:1 mixture of ${ }^{15} \mathrm{~N}$ - and spin- labeled chains. The spin label at the $\mathrm{N}$-terminal residue (Gly1) generated the strongest PRE, consistent with the funnel-like structure in which the $\mathrm{N}$-termini of the protomers at the funnel tip are very close $-\mathrm{N}$-termini of the neighboring chains within $14.8 \AA$ of each other (Fig. 2a). C-terminal residues 59-63 also show obvious PRE ( 0.6) because they are relatively close to the N-termini of neighboring chains. The spin label at His31 induced strong PREs $(0.5-0.6)$ near the C-terminal end (residues 36-42) of the $\mathrm{H} 2$ helix (Fig. 2b), also consistent with the interaction between the $\mathrm{H} 2$ segments from the neighboring chains shown by the hexamer structure. Ser44 is one of the most peripheral residues on the wider opening of the funnel, located in the flexible loop connecting the $\mathrm{H} 2$ and $\mathrm{H} 3$ helices. Spin label at Ser44 generated modest but obvious interchain PRE ( 0.7) for residues 41-45 (Fig. 2c). Finally, the spin label at Arg57 induced substantial inter-chain PRE ( 0.6) for residues 17-18, in good agreement with the proximity of Arg57 to the short joint (His17, Gly18) between H1 and H2 (Fig. 2d). Overall, the interchain PREs of p7(5a) in DPC is in agreement with the previously reported hexamer structure derived from inter-chain NOE data ${ }^{8}$ (see PRE distances in Fig. S5).

We then performed similar inter-chain PRE measurements for $\mathrm{p} 7(5 \mathrm{a})$ reconstituted in DMPC-DHPC bicelles with $q=0.55$ (Figs. S6-S7). In this case, mixed isotope and spin labeled chains were first reconstituted in bicelles, followed by labeling with MTSL and NiNTA affinity chromatography for removing excessive MTSLs bound to the bicelles. The spin label at His31 induced strong inter-chain PREs very similar to those observed for the micelle sample, although the PRE is on average stronger and a wider range of residues (3647) are affected (Fig. 3a). The slightly stronger inter-chain PREs were probably due to more intimate contacts between the $\mathrm{H} 2$ helical segments of the neighboring chains in the more membrane-like bicelles. The spin label at Arg57 also induced inter-chain PREs ( 0.6) similar to that detected in the micelle sample, e.g., residues around Gly18 were most affected (Fig. 3b). In addition to the joint between $\mathrm{H} 1$ and $\mathrm{H} 2$, however, the $\mathrm{H} 1$ in bicelles shows substantially stronger PREs than in micelles (Fig. 3b), possibly due to the more dynamic $\mathrm{H} 1$ helices in bicelles shown previously by NMR measurements of the apparent rotational correlation times ${ }^{14}$. The inter-chain PRE patterns generated by the two spin labels 
in bicelles are overall in agreement with the PREs induced by the same spin labels in micelles, indicating that the hexameric conformations in bicelles and micelles are similar.

Our SEC analysis of p7(5a) reconstituted under different detergent concentrations shows that the detergent:protein ratio has a profound influence on the oligomerization of p7(5a). Previous studies have already shown that, for certain small oligomeric membrane proteins, high detergent:protein ratio can cause membrane protein denaturation or misfolding ${ }^{15-17}$. More importantly, our SEC data address the major discrepancy between what appeared to be extremely similar NMR studies of HCV p7 by OuYang et a ${ }^{8}$ and Oestringer et al ${ }^{12}$. The two studies used the same 7 construct (p7(5a)), the same detergent (DPC), and recorded very similar 2D NMR spectra. But, the SEC result from Oestringer et al is clearly indicative of monomeric $\mathrm{p} 7$ in micelles, in sharp contrast to the hexameric species and inter-chain NOEs observed by OuYang et al. The results in Fig. 1 show that the very different detergent:protein ratios used during protein reconstitution were the cause of the discrepancy, providing an unambiguous resolution to the ongoing controversy of the $\mathrm{HCV}$ viroporin structure.

The strong dependence on detergent:protein ratio, however, suggests the fragility of the p7 oligomers in detergent micelles. Indeed, the inter-chain NOEs of p7(5a) in DPC micelles appeared weaker than those of transmembrane helix oligomers in bicelles ${ }^{18-20}$, and hence the intriguing architecture of the p7 oligomer revealed in DPC micelles has been challenged. Our site-specific PRE measurements of p7(5a) in DPC micelles and in DMPC-DHPC bicelles both show specific inter-chain PREs that are overall consistent with the published hexamer structure in micelles. Notably, the PREs generated by spin labels at different positions along the 6-fold axis all can be explained by the unusual fold of the protein, and this level of agreement is not possible if the protein were monomers generating PREs via non-specific aggregation.

Finally, earlier study of p7(5a) in DMPC-DHPC bicelles $(q=0.6)$ has already shown that the protein forms hexamers in bicelles ${ }^{14}$. The lipid and water NOE data from the same study are also consistent with the hexameric NMR structure, i.e., the H3 helices that wrap the channel periphery exhibited strong lipid NOEs whereas the pore-lining $\mathrm{H} 2$ helices did not. In the current study, our inter-chain PREs generated by spin labels at His31 and Arg57 further suggest that the mode of hexameric assembly (or arrangements of the helical segments) in bicelles is similar to that in micelles. The inter-chain PRE data are nevertheless semiquantitative. Detailed structural differences between the two systems would require comprehensive inter-chain NOE analysis.

\title{
Supplementary Material
}

Refer to Web version on PubMed Central for supplementary material.

\section{ACKNOWLEDGMENT}

\author{
We thank Dr. Fan Yang for PRE distance analysis, and Drs. Qingshan Fu and Alessandro Piai for suggestions on \\ PRE sample preparation and data analysis.
}

FUNDING SOURCES 
No competing financial interests have been declared. This work was supported by US NIH grants GM116898 and HL130143 (to J.J.C.) and CAS grant Key Research Program of Frontier Sciences QYZDB-SSW-SMC043 (to B.O.).

\title{
ABBERVIATIONS
}

\author{
DPC \\ Dodecylphosphocholine \\ DMPC \\ 1,2-Dimyristoylsn-Glycero-3-Phosphocholine \\ $\mathrm{DH}_{6} \mathrm{PC}$ \\ 1,2-Dihexanoyl-sn-Glycero-3-Phosphocholine \\ SEC \\ size-exclusion chromatography \\ PRE \\ Paramagnetic Relaxation Enhancement \\ MTSL \\ 1-oxyl-2,2,5,5-tetramethyl- $\Delta 3$-pyrroline-3-methyl \\ methanethiosulfonate
}

\section{REFERENCES}

(1). Griffin SD, Beales LP, Clarke DS, Worsfold O, Evans SD, Jaeger J, Harris MP, and Rowlands DJ (2003) FEBS Lett 535, 34-38. [PubMed: 12560074]

(2). Pavlovic D, Neville DC, Argaud O, Blumberg B, Dwek RA, Fischer WB, and Zitzmann N (2003) Proc Natl Acad Sci U S A 100, 6104-6108. [PubMed: 12719519]

(3). Premkumar A, Wilson L, Ewart GD, and Gage PW (2004) FEBS Lett 557, 99-103. [PubMed: 14741348]

(4). Montserret R, Saint N, Vanbelle C, Salvay AG, Simorre JP, Ebel C, Sapay N, Renisio JG, Bockmann A, Steinmann E, Pietschmann T, Dubuisson J, Chipot C, and Penin F (2010) J Biol Chem 285, 31446-31461. [PubMed: 20667830]

(5). Jones CT, Murray CL, Eastman DK, Tassello J, and Rice CM (2007) J Virol 81, 8374-8383. [PubMed: 17537845]

(6). Steinmann E, Penin F, Kallis S, Patel AH, Bartenschlager R, and Pietschmann T (2007) PLoS Pathog 3, e103. [PubMed: 17658949]

(7). Griffin S, Stgelais C, Owsianka AM, Patel AH, Rowlands D, and Harris M (2008) Hepatology 48, 1779-1790. [PubMed: 18828153]

(8). OuYang B, Xie S, Berardi MJ, Zhao X, Dev J, Yu W, Sun B, and Chou JJ (2013) Nature 498, 521525. [PubMed: 23739335]

(9). Luik P, Chew C, Aittoniemi J, Chang J, Wentworth P Jr., Dwek RA, Biggin PC, Venien-Bryan C, and Zitzmann N (2009) Proc Natl Acad Sci U S A 106, 12712-12716. [PubMed: 19590017]

(10). Chen W, OuYang B, and Chou JJ (2018) Nature 562, E19-E20. [PubMed: 30333581]

(11). Oestringer BP, Bolivar JH, Claridge JK, Almanea L, Chipot C, Dehez F, Holzmann N, Schnell JR, and Zitzmann N (2019) Scientific reports 9, 8383. [PubMed: 31182749]

(12). Oestringer BP, Bolivar JH, Hensen M, Claridge JK, Chipot C, Dehez F, Holzmann N, Zitzmann N, and Schnell JR (2018) Nature 562, E8-E18. [PubMed: 30333582]

(13). Dev J, Bruschweiler S, Ouyang B, and Chou JJ (2015) Journal of biomolecular NMR 61, 369378. [PubMed: 25724842]

(14). Chen W, Dev J, Mezhyrova J, Pan L, Piai A, and Chou JJ (2018) Structure 26, 627-634 e624. [PubMed: 29551287]

(15). Fisher LE, Engelman DM, and Sturgis JN (2003) Biophysical journal 85, 3097-3105. [PubMed: 14581210]

(16). Zhuang T, Jap BK, and Sanders CR (2011) Journal of the American Chemical Society 133, 20571-20580. [PubMed: 22084929] 
(17). Marinko JT, Huang H, Penn WD, Capra JA, Schlebach JP, and Sanders CR (2019) Chemical reviews 119, 5537-5606. [PubMed: 30608666]

(18). Fu Q, Fu TM, Cruz AC, Sengupta P, Thomas SK, Wang S, Siegel RM, Wu H, and Chou JJ (2016) Mol Cell 61, 602-613. [PubMed: 26853147]

(19). Dev J, Park D, Fu Q, Chen J, Ha HJ, Ghantous F, Herrmann T, Chang W, Liu Z, Frey G, Seaman MS, Chen B, and Chou JJ (2016) Science 353, 172-175. [PubMed: 27338706]

(20). Pan L, Fu TM, Zhao W, Zhao L, Chen W, Qiu C, Liu W, Liu Z, Piai A, Fu Q, Chen S, Wu H, and Chou JJ (2019) Cell 176, 1477-1489 e1414. [PubMed: 30827683] 


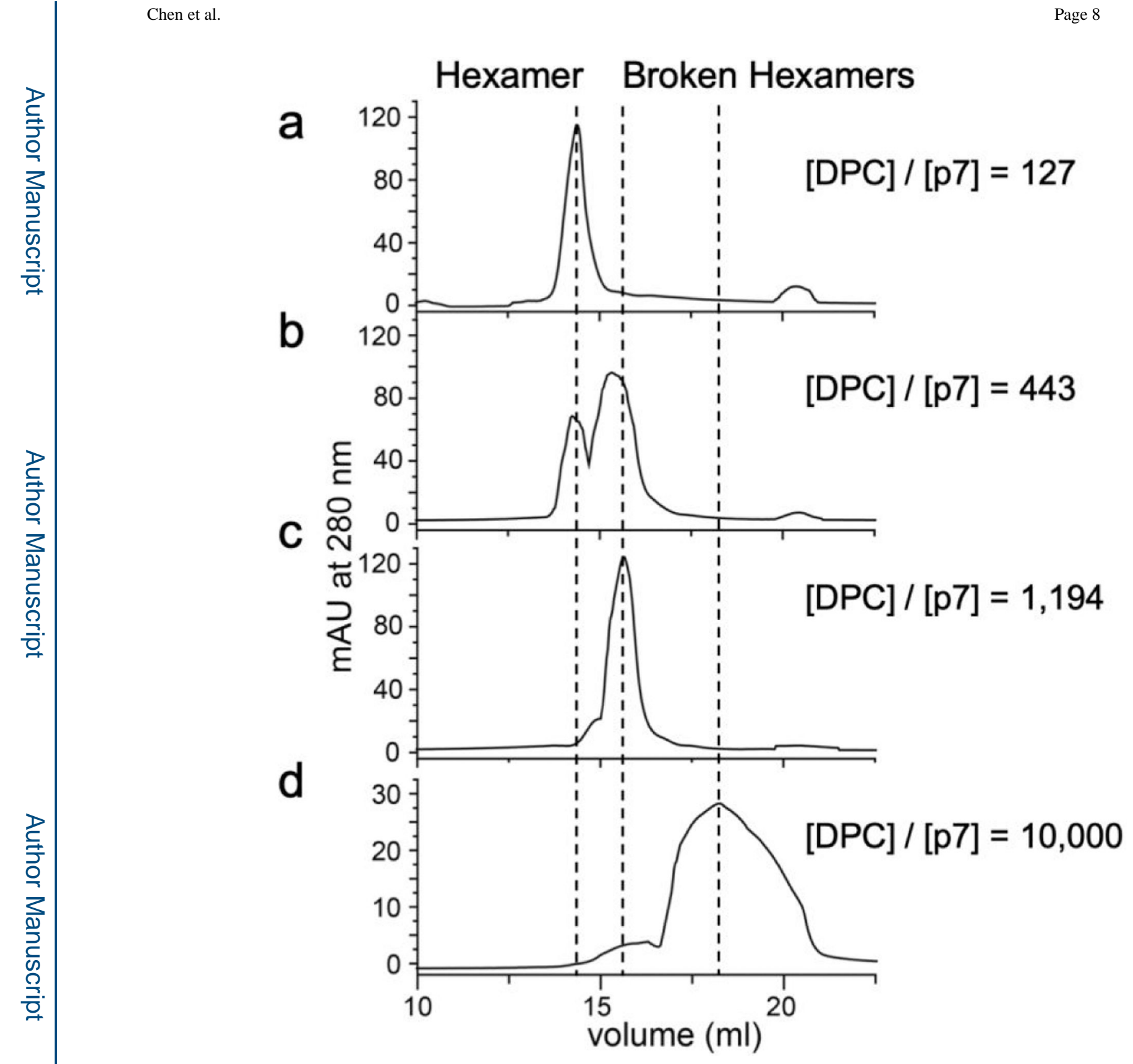

FIGURE 1.

Size-exclusion chromatography (SEC) profiles of $\mathrm{p} 7(5 \mathrm{a})$ reconstituted in different amounts of DPC. a-d) SEC elution profile from the superdex 200 increase 10/300 GL column of p7(5a) reconstituted initially at DPC:protein ratio of 127 (a), 443 (b), 1,194 (c), and 10,000 (d). [p7] $=0.35 \mathrm{mM}$ in a-c and $0.1 \mathrm{mM}$ in d. [DPC] of reconstituted samples after dialysis were determined by ${ }^{1} \mathrm{H}$ NMR. 

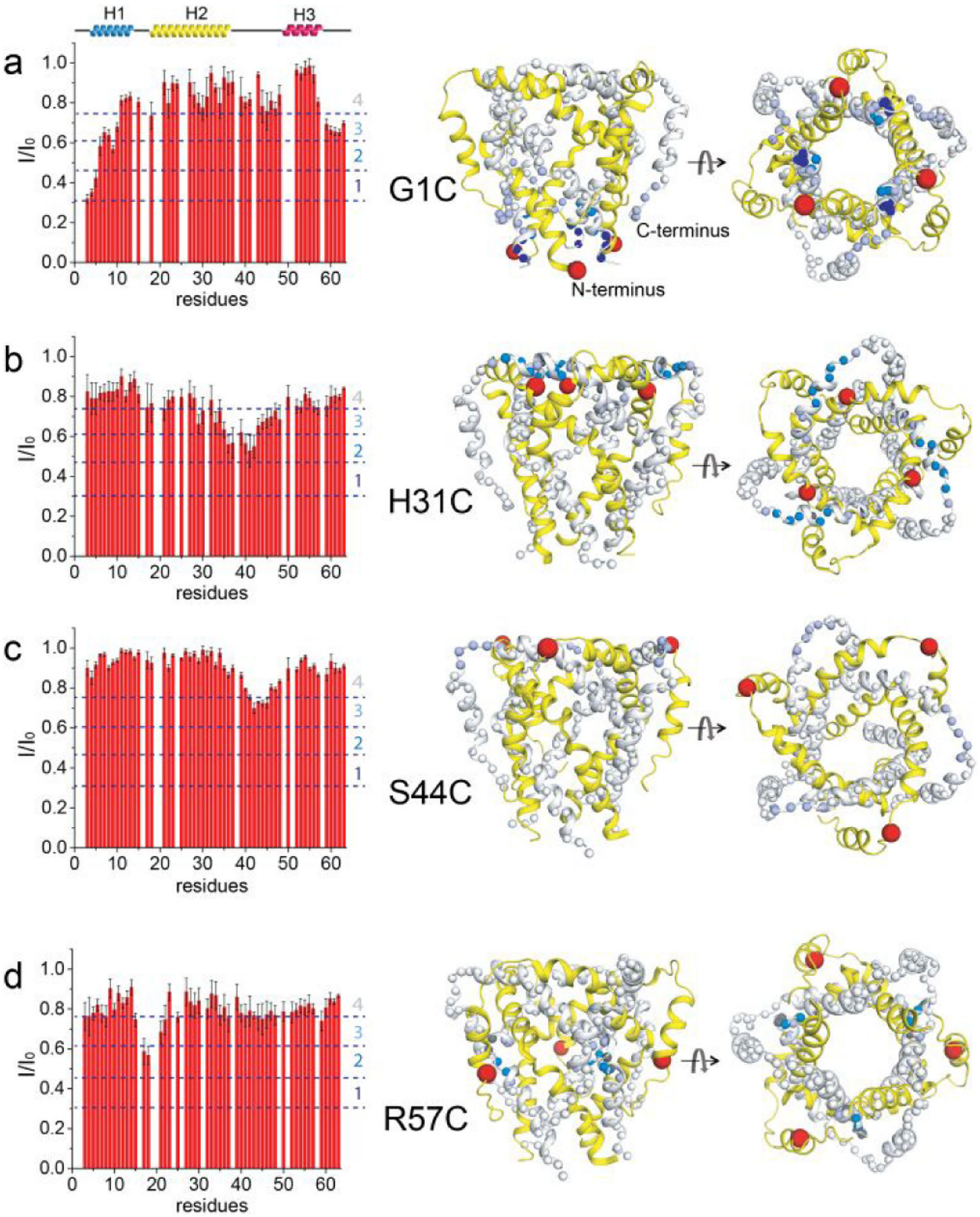

FIGURE 2.

Inter-chain PREs of p7(5a) samples in DPC micelles. The samples consist of 1:1 ratio of $\left({ }^{15} \mathrm{~N}, \sim 80 \%{ }^{2} \mathrm{H}\right)$ labeled protein and unlabeled protein with MTSL labeled at residue position 1 (a), 31 (b), $44(\mathbf{c})$, and 57 (d), reconstituted in DPC micelles. Left: Residuespecific PREs, defined as the ratio of intensity before (I) and after $\left(\mathrm{I}_{0}\right)$ reducing with ascorbate. Regions between the dashed lines define different PRE ranges. Right: Structural views (PDB ID: 2M6X) of backbone amide protons (spheres) that show strong PRE. Ribbon representations of the ${ }^{15} \mathrm{~N}$ and MTSL labeled strands are shown in gray and yellow, respectively (note the mixture is purely random). The MTSL position is indicated by the red sphere. The amide protons are colored according to the PRE ranges defined on the left. 

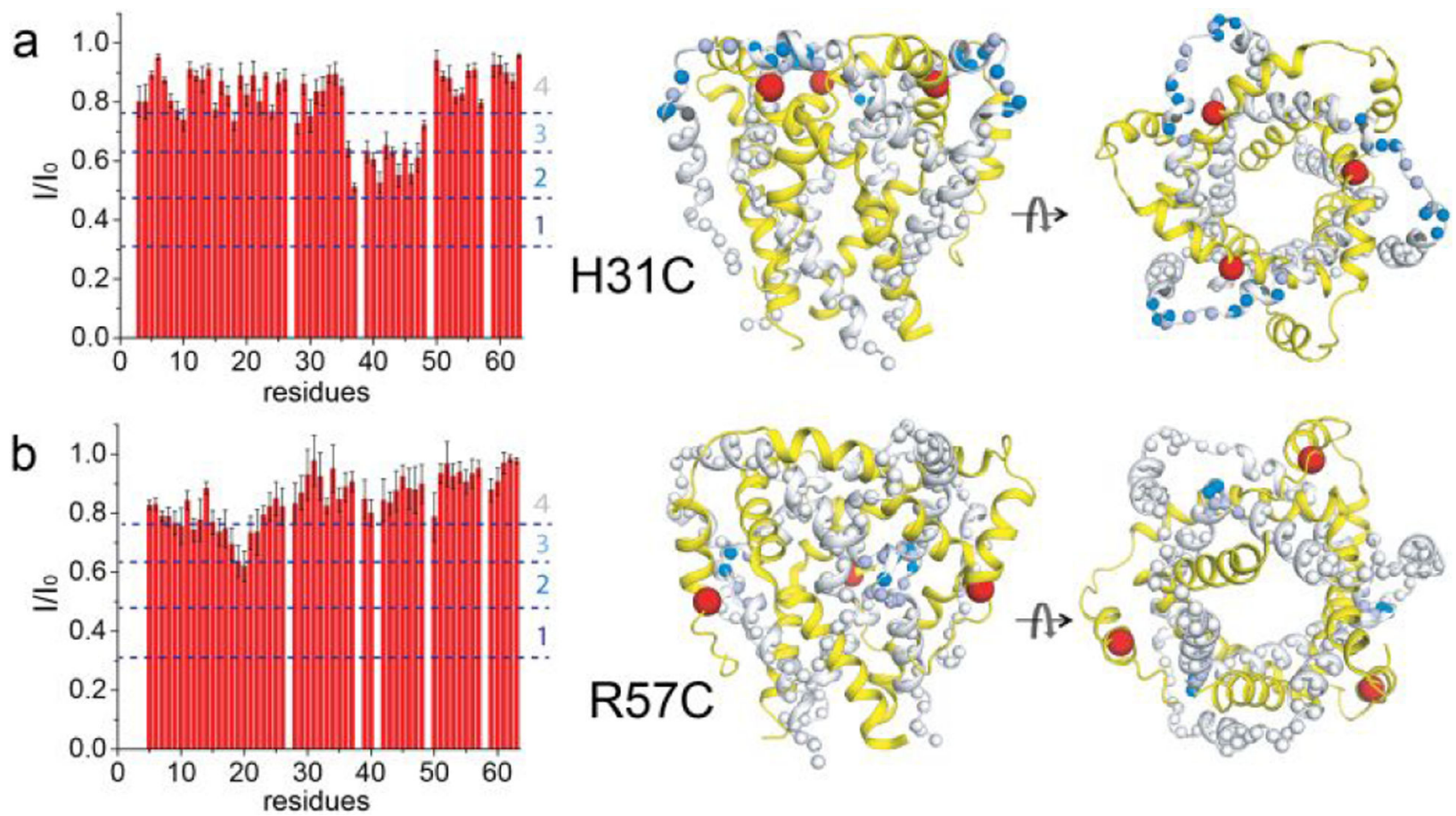

FIGURE 3.

Inter-chain PREs of p7(5a) samples in bicelles. The samples consist of 1:1 ratio of $\left({ }^{15} \mathrm{~N}\right.$, $\sim 80 \%{ }^{2} \mathrm{H}$ ) labeled protein and unlabeled protein with MTSL labeled at residue positions 31 (a) and 57 (b), reconstituted in DMPC-DHPC bicelles with $q=0.55$. Presentations in the left and right panels are the same as in Fig. 2. The MTSL position is indicated by the red sphere. The amide protons are colored according to the PRE ranges defined on the left. 